\title{
BMJ Open Exploring an adapted Risk Behaviour Diagnosis Scale among Indigenous Australian women who had experiences of smoking during pregnancy: a cross- sectional survey in regional New South Wales, Australia
}

\author{
Gillian Sandra Gould, ${ }^{1}$ Michelle Bovill, ${ }^{1}$ Simon Chiu, ${ }^{2}$ Billie Bonevski, ${ }^{1}$ \\ Christopher Oldmeadow ${ }^{2}$
}

To cite: Gould GS, Bovill M, Chiu S, et al. Exploring an adapted Risk Behaviour Diagnosis Scale among Indigenous Australian women who had experiences of smoking during pregnancy: a cross-sectional survey in regional New South Wales, Australia. BMJ Open 2017;0:e015054. doi:10.1136/ bmjopen-2016-015054

- Prepublication history and additional material are available. To view please visit the journal (http://dx.doi.org/10.1136/ bmjopen-2016-015054).

Received 8 November 2016 Revised 5 February 2017 Accepted 1 March 2017

\section{CrossMark}

${ }^{1}$ Centre for Brain and Mental Health Research, School of Medicine and Public Health, The University of Newcastle, University Drive, Callaghan, New South Wales, Australia ${ }^{2}$ Clinical Research Design, Information Technology and Statistical Support, Hunter Medical Research Institute, 1/1 Kookaburra Circuit, New South Wales, Australia

\section{Correspondence to} Associate Professor Gillian Sandra Gould; gillian.gould@newcastle.edu.au

\section{ABSTRACT}

Objectives Explore Aboriginal women's responses to an adapted Risk Behaviour Diagnosis (RBD) Scale about smoking in pregnancy.

Methods and design An Aboriginal researcher interviewed women and completed a cross-sectional survey including 20 Likert scales.

Setting Aboriginal Community Controlled Health Services, community groups and playgroups and Aboriginal Maternity Services in regional New South Wales, Australia. Participants Aboriginal women $(n=20)$ who were pregnant or gave birth in the preceding 18 months; included if they had experiences of smoking or quitting during pregnancy.

Primary and secondary outcome measures Primary outcomes: RBD constructs of perceived threat and perceived efficacy, dichotomised into high versus low. Women who had quit smoking, answered retrospectively. Secondary outcome measures: smoking status, intentions to quit smoking (danger control), protection responses (to babies/others) and fear control responses (denial/refutation). Scales were assessed for internal consistency. A chart plotted responses from low to high efficacy and low to high threat.

Results RBD Scales had moderate-to-good consistency (0.67-0.89 Cronbach's alpha). Nine women had quit and 11 were smoking; 6 currently pregnant and 14 recently pregnant. Mean efficacy level $3.9(\mathrm{SD}=0.7)$; mean threat 4.3 ( $S D=0.7)$. On inspection, a scatter plot revealed a cluster of 12 women in the high efficacy-high threat quadrantof these 11 had quit or had a high intention of quitting. Conversely, a group with low threat-low efficacy (5 women) were all smokers and had high fear control responses: of these, 4 had low protection responses. Pregnant women had a non-significant trend for higher threat and lower efficacy, than those previously pregnant.

Conclusion Findings were consistent with a previously validated RBD Scale showing Aboriginal smokers with high efficacy-high threat had greater intentions to quit smoking. The RBD Scale could have diagnostic potential to tailor health messages. Longitudinal research required with a larger sample to explore associations with the RBD Scale and quitting.
Strengths and limitations of this study

- The RBD Scale was adapted for pregnancy from a scale previously validated in Aboriginal smokers of reproductive age.

- Having an Aboriginal woman conduct the survey face-to-face in an environment of the participant's choosing fostered feasibility and acceptability of the survey.

- Sample was not intended to be a representative sample of the population, but purposively recruited to encompass a range of views.

- The small sample and dichotomising the high-low categories with median cut points restricted the findings: a larger study is required for determining clinically meaningful or predictive cut points.

- Women who had quit smoking were asked to rate their agreement with attitudinal statements retrospectively.

\section{INTRODUCTION}

Smoking prevalence is generally decreasing in most population groups within the high-income countries. It is becoming apparent that some high priority populations are being left behind in this positive trend. Tobacco smoking prevalence among Indigenous populations in high-income countries has been persistently much higher than their non-Indigenous counterparts. ${ }^{1}$ Women of reproductive age are vulnerable to additional effects from smoking if they become pregnant: on their birth outcomes, and the health of their newborn babies. ${ }^{2}$ Smoking is well-recognised as the single most important remediable risk factor in pregnancy for maternal and child health. ${ }^{2}$ Children born to mothers who smoke have long-term detrimental effects into adulthood. ${ }^{3}$ 
Smoking rates have been slow to decline in pregnant Indigenous Australians. Nationally, $47 \%$ of Indigenous Australian women smoke during pregnancy compared with $13 \%$ of their general population counterparts. ${ }^{4}$ Smoking cessation rates are reported as 3\%-4\% of Indigenous Australian women in the first half of pregnancy, ${ }^{56}$ and $11 \%$ in the second half of pregnancy compared with $25 \%$ in non-Indigenous pregnant women. ${ }^{4}$ Many factors have been put forward as barriers to explain this slower decline. These include historical factors relating to colonisation and dispossession, social norms of smoking in Indigenous communities, life stressors, lack of salience of health messages for Indigenous women, poor access to services and late antenatal presentation. ${ }^{78}$ Indigenous women commonly reduce consumption in pregnancy, and are aware that smoking is not healthy for the baby. ${ }^{910}$

In many populations where behaviour change is challenging, such as in sexually active youth and substance users, the Risk Behaviour Diagnosis (RBD) Scale has been found to be helpful to predict behavioural attitudes governing change, and inform the development of targeted messages and interventions. ${ }^{11}$ These have ranged from sexual health to smoking. ${ }^{12}$ In 2014, Gould et alvalidated the RBD Scale in a cohort of Indigenous Australian smokers of reproductive age and found that the scale, measuring perceived levels of threat and efficacy was correlated with intentions to quit smoking and creating smoke-free homes. ${ }^{14}$ The scale has not been used as far as we are aware in a pregnant population of smokers.

\section{RBD Scale}

The RBD Scale is based on the Extended Parallel Process Model (EPPM).${ }^{15}$ The EPPM proposes that the constructs of perceived efficacy (response efficacy and self-efficacy) and perceived threat (susceptibility to and severity of threat) have a relation to actions taken to avert the danger from the threat (danger control) or alternatively a defensive or avoidant response (fear control). Figure 1 shows the expected responses depending on whether threat is high or low according to the EPPM.

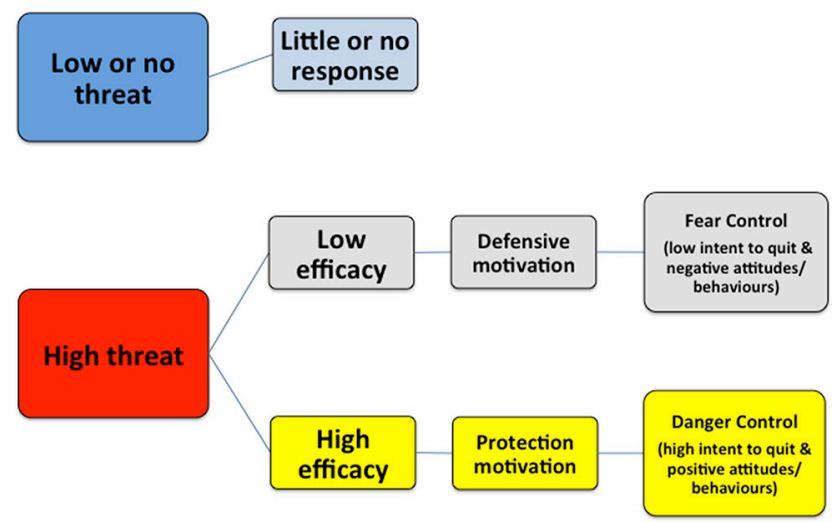

Figure 1 The extended parallel process model and expected responses to threat and efficacy levels ${ }^{16}$ (adapted from). ${ }^{26}$
The aim of this study was to explore the responses of a sample of Indigenous Australian women who had recent experiences of smoking or quitting during pregnancy, to an adapted RBD Scale. The adapted RBD Scale was assessed for face validity, feasibility of use with maternal smokers and ex-smokers, internal consistency and preliminary outcomes related to demographic characteristics of the participants. This study was additional to a qualitative inquiry into smoking or quitting during pregnancy.

\section{METHODS}

\section{Study setting and recruitment}

In a regional area of New South Wales (NSW), 20 Aboriginal women completed an online cross-sectional survey at the commencement of an interview with an Aboriginal Research Assistant (ARA), from August 2015 until April 2016. The women were purposively recruited for a qualitative study: the number determined by saturation of the qualitative themes. Participants were from local Aboriginal Community Controlled Health Services, Aboriginal Community groups and playgroups and Aboriginal Maternal and Infant Health Services. Staff from services advertised the study to women through a flyer and by personal approach. Some women were contacted during a telephone evaluation of a local Aboriginal targeted maternal smoking cessation programme. The ARA also recruited women from her Aboriginal community network. Inclusion criteria were women aged 16 years or over, either pregnant at the time or who had given birth within 18 months. They needed to have had experiences of smoking or quitting during pregnancy.

Nineteen women were individually interviewed face-toface: one interview was via telephone. The locations were chosen by the participant; a health or community centre $(n=7)$, private home $(n=7)$, playgroup or other $(n=5)$.

Human Research Ethics Committees from Hunter New England Local Health District, University of Newcastle, and Aboriginal Health and Medical Research Council NSW approved the study.

\section{Procedures}

The ARA explained the purpose of the study and gained informed consent by asking women to read, or have a standard consent form read to them, and making sure it was understood. Women completed a short online survey aided by the ARA. The data were collected through a secure Qualtrics online website. Face validity of the questions was qualitatively assessed as part of an interactive process between each woman and the interviewer, that is, by observing whether the questions were understood and self-explanatory-which in the majority of cases they were.

\section{Survey instrument}

The survey questions encompassed demographic details, smoking behaviours, intentions to quit smoking and the RBD Scale. Demographic questions included: age, 
education level (primary school to year 9, year 10-11, trade certificate, current tertiary student, undergraduate qualification), a government concession (healthcare) card $(\mathrm{Y} / \mathrm{N})$, number of live children $(0,1-2,3$ or more), a child living at home $(\mathrm{Y} / \mathrm{N})$, pregnant status $(\mathrm{Y} / \mathrm{N})$. Pregnant women were asked how far into their pregnancy they were (first 3 months, 3-6 months, $>6$ months); those not pregnant were asked when they gave birth $(<4$ weeks, 5-12 weeks, 3 months-1 year, $>1$ year, other).

Women were asked about their smoking status $(\mathrm{Y} / \mathrm{N})$, what they smoke (normal cigarettes, hand-rolled, pipe/ cigars, other), how often they smoke (every day, most days, occasional), household smoking rules (no smoking inside nor on veranda (porch)/just outside; smoking only on veranda/outside; smoking in some rooms inside; smoking anywhere); number of smokers in household (0, $1,2-3,>3)$. Two standard questions were asked comprising the Heaviness of Smoking Index (cigarettes per day and time to first cigarette). Smokers were asked about their strength and frequency of urges to smoke. For frequency of urges they were asked to indicate: How much of the time have you felt the urge to smoke in the last 24 hours? Not at all; a little of the time; some of the time; a lot of the time; almost all the time or all the time. For strength of urges the question was: In general, how strong are your urges to smoke (in the last 24 hours)? No urges; slight; moderate; strong; very strong; extremely strong. Smokers were exposed to questions about their intentions to quit (danger control response questions in item 7 below).

\section{RBD Scale}

The RBD Scale is a set of questions designed as metrics to measure perceived efficacy and perceived threat. The RBD Scale is made up of four subscales; response efficacy, self-efficacy, susceptibility to threat, severity of threat and two additional related scales to measure fear control and danger control responses. Gould added another series of questions on protection responses, for Indigenous Australians. ${ }^{16}$ The RBD Scale was validated in 121 Indigenous Australians from NSW of reproductive age. ${ }^{14}$

Questions from the validated RBD Scale ${ }^{14}$ were modified by GG to reflect the pregnant and postpartum context. This was done with the implication of effects towards the child for the threats, response efficacy and fear control. An example is 'giving up smoking helps avoid serious sickness or disease for babies'. The scales for self-efficacy, danger control responses and protection were the same as the previously validated scale. The questions are detailed in Box 1.

Participants completed two possible versions of the survey dependent on the response to "Do you currently smoke?" This question diverged participants into either present tense for smokers or past tense for non-smokers.

Popova suggest the results of the RBD Scale can be dichotomised (high-low) and arranged into four quadrants as follows, with associations with four corresponding different types of behaviours to health messages ${ }^{1718}$ :

The RBD quadrants are defined as:
Box 1: Risk Behaviour Diagnosis Scale questions explored in 20 Aboriginal women

1. Responses efficacy: 5-point Likert scale (strongly disagree-strongly agree)

a. Stopping smoking prevents serious sickness or disease for a baby

b. Giving up smoking helps avoid serious sickness or disease for babies

c. If I stop smoking my baby is less likely to get a serious sickness or disease

2. Self-efficacy: 5-point Likert scale (strongly disagree-strongly agree)

a. I am confident I can stop smoking

b. I am able to stop smoking

c. It is easy to stop smoking

3. Susceptibility to threat: 5-point Likert scale (strongly disagreestrongly agree)

a. It is likely that my baby will get ill from my smoking

b. Smoking could possibly affect my baby's health

c. I believe my baby is seriously at risk of getting ill from smoking

4. Severity of threat: 5-point Likert scale (strongly disagree-strongly agree)

a. Smoking is harmful to the health of a baby

b. Smoking by pregnant mums can severely affect the health of babies

c. The health effects of mum's smoking are of serious concern for a baby

5. Protection responses: 5-point Likert scale (strongly disagreestrongly agree)

a. It is better if pregnant women do not smoke

b. It is better if partners of pregnant women quit smoking

c. It is better if adults do not smoke around children and babies

d. It is better if Aboriginal or Torres Strait Islander people do not smoke at all

6. Fear control responses: 5-point Likert scale (strongly disagreestrongly agree)

a. I prefer not to think about the health risks of smoking for my baby

b. The risks of smoking to a baby are overdone or overblown

c. I do not personally believe that smoking is going to affect my baby's health

d. The risks of smoking for babies are untrue or manipulated

7. Danger control responses: 4-point Likert scale (very unlikely-very likely). How likely is it that in the next 3 months you will:

a. Quit smoking completely and permanently

b. Reduce the number of cigarettes you smoke in a day

c. Talk to a friend or family about quitting smoking

d. Seek professional help to quit smoking

e. Enroll in a smoking cessation programme (if available at minimal cost)

Q1: High threat-high efficacy: responsive behaviour

Q2: High threat-low efficacy: avoidant behaviour

Q3: Low threat-high efficacy: proactive behaviour

Q4: Low threat-low efficacy: indifferent behaviour

\section{Analysis}

All statistical analyses were programmed using SAS V.9.4 (SAS Institute, Cary, North Carolina, USA).

The scales had acceptable face validity when the instrument was administered. The ARA reported that the 
questions were understood when read out to the women, although they seemed rather repetitive. Cronbach's alpha and item-total correlations were used to assess the internal consistency of the scales. The divergent results (smokers vs non-smokers) were pooled into overall responses to the RBD under the assumption that the change in tense did not affect results and that the two questionnaires were comparable.

The scores for each of the subscales (listed in Box 1) were calculated for each participant as the summation of their Likert-scale responses within the subscale, divided by the number of items in the subscale.

Additionally, total perceived efficacy was created from the scale items in self-efficacy and response efficacy. Similarly, total perceived threat was produced from the items in the susceptibility to threat and severity of threat. Total perceived efficacy, total perceived threat, protection response, fear control response and danger control response (intentions to quit smoking) subscales were split at the median to create a dichotomous binary variable where responds greater or equal to the median were classed 'High' and responses lower than the median were classed as 'Low'. Since the danger control responses only applied to smokers, danger control category was grouped into non-smoker, high intent and low intent to quit smoking.

Associations between demographic variables, based on clinical relevance, were investigated comparing smokers against ex-smokers. Fisher's exact test was used to assess associations between RBD quadrants with:

a. Demographics: smoking status, household smoking bans, pregnant status

b. Additional dichotomous scales: protection response, fear control responses and danger control response.

Scatter plots were used to look for outlying cases to validate and justify associations.

\section{RESULTS}

Table 1 shows the demographics for the 20 participants overall and by smoking status. There were no statistically significant associations between the demographics and smoking status.

\section{Internal consistency}

There were no missing data for the survey responses to the RBD items. Coefficients of the RBD metrics ranged from 0.67 to 0.92 . Cronbach's alpha of 0.7 or over is usually acceptable. ${ }^{19}$ The pairwise correlation between measures ranged from just under 0.7 to excellent $(\geq 0.9)$.

For the total perceived efficacy subscale, there was a low item-total correlation ( $r)$ for question 2c $(r=0.30)$. The removal of this item made only a small difference to the internal consistency, so was retained in the analysis (a similar finding to the original validation). The same conclusion was made with regard to total perceived threat

\begin{tabular}{|c|c|c|c|c|}
\hline Variable & Smoker $(n=11)$ & Non-smoker $(n=9)$ & Total $(n=20)$ & Fisher's exact test \\
\hline \multicolumn{5}{|l|}{ Age } \\
\hline $16-24$ & $3(27 \%)$ & $3(33 \%)$ & $6(30 \%)$ & 0.668 \\
\hline $25-34$ & $5(45 \%)$ & $5(56 \%)$ & $10(50 \%)$ & \\
\hline $35-45$ & $3(27 \%)$ & $1(11 \%)$ & $4(20 \%)$ & \\
\hline \multicolumn{5}{|l|}{ Highest level of education } \\
\hline Primary up to year 9 & $4(36 \%)$ & $1(11 \%)$ & $5(25 \%)$ & \\
\hline Year $10-11$ & $3(27 \%)$ & $5(56 \%)$ & $8(40 \%)$ & 0.345 \\
\hline Trade certificate & $1(9.1 \%)$ & $2(22 \%)$ & $3(15 \%)$ & \\
\hline Current student & $2(18 \%)$ & & $2(10 \%)$ & \\
\hline Undergraduate & $1(9.1 \%)$ & $1(11 \%)$ & $2(10 \%)$ & \\
\hline \multicolumn{5}{|l|}{ No. of children } \\
\hline None & $1(9.1 \%)$ & & $1(5.0 \%)$ & 0.236 \\
\hline $1-2$ & $4(36 \%)$ & $6(67 \%)$ & $10(50 \%)$ & \\
\hline 3 or more & $3(27 \%)$ & $3(33 \%)$ & $6(30 \%)$ & \\
\hline Other & $3(27 \%)$ & & $3(15 \%)$ & \\
\hline \multicolumn{5}{|l|}{ Home smoking ban } \\
\hline No smoking inside or outside & $4(36 \%)$ & $2(22 \%)$ & $6(30 \%)$ & 0.492 \\
\hline Smoking only outside & $7(64 \%)$ & $7(78 \%)$ & $14(70 \%)$ & \\
\hline \multicolumn{5}{|l|}{ Currently pregnant? } \\
\hline Yes & $2(18 \%)$ & $4(44 \%)$ & $6(30 \%)$ & 0.202 \\
\hline No & $9(82 \%)$ & $5(56 \%)$ & 14 (70\%) & \\
\hline
\end{tabular}


Table 2 Dichotomous* total perceived threat and efficacy in 20 Aboriginal women

\begin{tabular}{|c|c|c|c|c|}
\hline Variable & & n (\%) & Median (min, max) & $\begin{array}{l}\text { Cronbach's Alpha } \\
\text { (a) }\end{array}$ \\
\hline \multirow[t]{2}{*}{ Total perceived efficacy } & Low & $9(45 \%)$ & $4.0(2.7,4,8)$ & 0.8 \\
\hline & High & $11(55 \%)$ & & \\
\hline \multirow[t]{2}{*}{ Total perceived threat } & Low & $8(40 \%)$ & $4.3(2.0,5.0)$ & 0.89 \\
\hline & High & $12(60 \%)$ & & \\
\hline \multirow[t]{2}{*}{ Protection response } & Low & $8(40 \%)$ & $4.3(2.8,5.0)$ & 0.53 \\
\hline & High & $12(60 \%)$ & & \\
\hline \multirow[t]{2}{*}{ Fear control response } & Low & $9(45 \%)$ & $2.0(1.0,4.3)$ & 0.78 \\
\hline & High & $11(55 \%)$ & & \\
\hline \multirow[t]{2}{*}{ Danger control response (smokers only) } & Low & $5(45 \%)$ & $2.4(1.4,3.6)$ & 0.85 \\
\hline & High & $6(55 \%)$ & & \\
\hline
\end{tabular}

*Low classed as less than median, High classed as greater or equal to median.

and fear control response; question $4 \mathrm{~b}(\mathrm{r}=0.51)$ and questions $6 \mathrm{a}(\mathrm{r}=0.43)$ also had low item-total correlations. However, their exclusion minimally increased constancy.

The Cronbach's alpha for protection response was poor $(\alpha=0.53)$, a very low item-total correlation was observed for question $5 \mathrm{~b}(\mathrm{r}=-0.04)$. Since the RBD is a validated measure and for the reasons above, protection response was left unchanged. Question 5b 'it is better if partners of pregnant women quit smoking' may have a different salience when women are close to pregnancy themselves, compared to the responses from the more general sample previously tested comprising both genders. ${ }^{14}$ In this case, 17 out of 20 of these pregnant or recently pregnant women agreed or strongly agreed that partners should quit smoking.

\section{Analysis of quadrants}

Table 2 shows the frequency of the participant allocations into the 'High' and 'Low' categories for perceived threat and perceived efficacy. Generally, since the median was chosen as the cut point it was expected that the distributions into the categories would be half, the members that scored the median value are allocated to the 'High' category, this favours the 'High' dichotomy.

Figure 2 shows a scatter plot of the 20 respondents. The theory behind the grouping of the EPPM into the four quadrants suggests that the members of quadrant one hold responsive attitudes towards quitting; conversely, members that fall into quadrant four have indifferent attitudes. ${ }^{17} 18$ Figure 2 shows that there are members that deviated from the expected EPPM theory: there are four cases of current smokers in quadrant one, one pregnant and three post partum. On examining the qualitative data to gain an explanation, the analysis revealed that the three postpartum participants had in common that they reported suffering from depression. However, these three smokers had high intentions to quit. Similarly, there were two postpartum smokers (one of which cited depression) with relatively high threat mean scores allocated to the avoidant behaviour quadrant (Q2). The woman citing depression in Q2 had conversely a low intention to quit smoking.

Without a larger sample, it is difficult to determine the causality of these anomalies. At a descriptive level, we can conclude that ex-smokers have a mean threat score of 4 or greater. Out of the seven smokers with threat mean scores about 4, five have a high intention to quit and four have cited depression.

\section{DISCUSSION}

\section{Principal findings}

This exploratory quantitative description of the attitudes of 20 Aboriginal women was in addition to a qualitative inquiry about their experiences of smoking and quitting in pregnancy. The analysis set out to investigate how an adapted RBD Scale performs in the context of pregnant Indigenous females as an extension of previous work validating the use of the RBD in regional NSW. ${ }^{14}$

The RBD Scales had moderate-to-good consistency (0.67-0.89 Cronbach's alpha). Mean efficacy level was 3.9

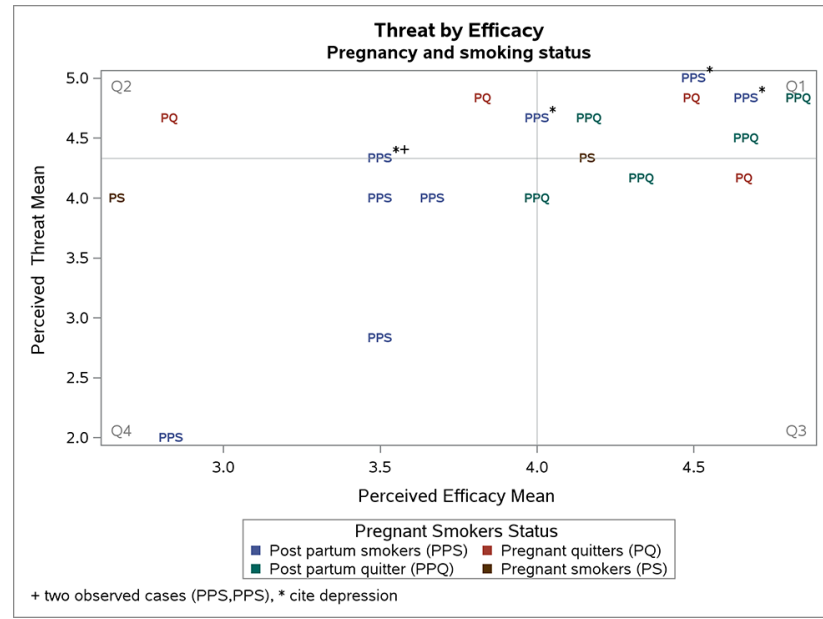

Figure 2 Scatter plot of total threat by total efficacy in 20 Aboriginal women. 
$(\mathrm{SD}=0.7)$; mean threat was $4.3(\mathrm{SD}=0.7)$. On inspection, a scatter plot revealed a cluster of 12 women in the high efficacy-high threat quadrant: of these, 11 had quit or had a high intention of quitting. Conversely, a group with low threat-low efficacy (five women) were all smokers and had high fear control responses: of these, four had low protection responses. Pregnant women had a non-significant trend for higher threat and lower efficacy, than those previously pregnant.

\section{Strengths and weaknesses of the study}

The small sample of data restricts the variety of statistical techniques. The use of median cut points to dichotomise the high-low categories creates an artificial push away from quadrant 4 (indifferent attitudes), but has been used previously. ${ }^{14}$ Further studies would be required to determine potentially clinically meaningful or predictive cut points. ${ }^{18}$ The sample was not intended to be a representative sample of the population, but purposively recruited to encompass a range of views, therefore, results may not be generalisable to the broader Indigenous population.

The strength of the study was having an Aboriginal woman conduct the survey face-to-face in an environment of the participant's choosing, to foster feasibility and acceptability of the use of the survey. Also using the RBD Scale previously validated in Aboriginal smokers of reproductive age, facilitated the adaptation of the Scale for Aboriginal pregnancy.

\section{Strengths and weaknesses in relation to other studies}

Low self-efficacy for quitting has been previously noted in populations of pregnant smokers, including Indigenous women. ${ }^{72021}$ Similarly, the perception of smoking risks during pregnancy have been well described. ${ }^{102} 23$ However, other studies as far as we know have not measured the constructs of efficacy in relation to threat perceptions in maternal smokers.

The RBD Scale has been used only once before in a population of Aboriginal smokers, as far as we know. These findings in a group of 20 pregnant and recently pregnant Indigenous Australian women are consistent with the previously validated RBD Scale, showing that Aboriginal smokers with high efficacy-high threat had more intentions to quit smoking. ${ }^{14}$ In comparison, strength of this study was that some women had already quit smoking, leading to preliminary analysis of current smokers versus those who had quit, whereas in the previous study all participants were still smoking. Some pregnancy-specific differences were also noted (eg, a trend towards higher threat and lower efficacy), requiring a larger study to validate the RBD Scale in pregnancy.

\section{Meaning of the study}

In the climate that little is known about how to motivate pregnant Indigenous women to quit smoking and maintain abstinence post partum, this study adds insight. High threat and high efficacy states show a promising trend towards abstinence and/or a high intention to quit, whereas women with low efficacy and low threat demonstrated that they were maintaining smoking, and low intention to quit in the near future. Perceived efficacy and threat levels are potentially changeable. When risk and efficacy are made salient, people's risk perception can guide their subsequent actions. ${ }^{18}$ The prevailing threat messages that pregnant women are exposed to via the media may seem too threatening and engender avoidance, ${ }^{24}$ especially if women have low efficacy. It has been noted in pregnancy that risk messages need to be carefully pitched and supported by strategies to build efficacy. ${ }^{9}$ Building efficacy can include a range of measures: self-efficacy can be appropriately supported in consultation with healthcare professionals, or in cessation groups and by providing assistance with nicotine replacement therapy, support for the woman in her psychosocial context and involving family as supporters. $^{25}$ Response efficacy is also important to build-helping the women feel optimistic that quitting is worth it, and is likely to improve the outlook for both her and her baby.

\section{Implications}

Assessing the feasibility and acceptability of use of the adapted RBD Scale in Aboriginal women is an important initial step before considering its use as a prospective tool. The RBD Scale could have diagnostic potential in the tailoring of health messages and supportive strategies. ${ }^{26}$ Health messages delivered in clinical settings could be selected according to the relative levels of women's self-efficacy versus threat. Further research is required using a larger sample of pregnant Indigenous women, and preferably prospectively following them over two time points to see if the RBD Scale has any association with quitting behaviours. Other confounding factors such as depression may need consideration.

Acknowledgements We thank the services and communities in Hunter New England that assisted with recruitment including: Aboriginal Community Controlled Health Services, Aboriginal Maternal and Infant Health Services, Quit For New Life programme and Gomeroi Gaaynggal centre. We especially thank the women who generously participated.

Contributors GG conceived the study, designed the survey and the methods, prepared the data, contributed to the analysis and data interpretation and wrote the paper. MB conducted the data collection, and contributed to the cultural interpretation of findings and final paper. SC conducted the statistical analysis and wrote the preliminary findings. BB oversaw the study, contributed to the interpretation and critically reviewed the final paper. CO oversaw the statistical analysis, interpretation and writing of the results, and contributed to the final paper.

Competing interests None declared.

Ethics approval University of Newcastle HREC and Aboriginal Health and Medical Research Council HREC.

Provenance and peer review Not commissioned; externally peer reviewed.

Data sharing statement Indigenous protocols may limit the sharing of data for this paper.

Open Access This is an Open Access article distributed in accordance with the Creative Commons Attribution Non Commercial (CC BY-NC 4.0) license, which permits others to distribute, remix, adapt, build upon this work non-commercially, and license their derivative works on different terms, provided the original work is 
properly cited and the use is non-commercial. See: http://creativecommons.org/ licenses/by-nc/4.0/

(C) Article author(s) (or their employer(s) unless otherwise stated in the text of the article) 2017. All rights reserved. No commercial use is permitted unless otherwise expressly granted.

\section{REFERENCES}

1. Carson KV, Robertson TA, Brinn MP, et al. Tobacco use, cessation and prevention for Indigenous populations: Review of current evidence and future perspectives. Jackson OT EK, ed. Health disparities: epidemiology, racial/Ethnic and socioeconomic risk factors and strategies for elimination. public health in the 21st century. New York: Nova Science Publishers, 2013:1-38. p.

2. Hofhuis W, de Jongste JC, Merkus PJ. Adverse health effects of prenatal and postnatal tobacco smoke exposure on children. Arch Dis Child 2003;88:1086-90.

3. Knopik VS, Maccani MA, Francazio S, et al. The epigenetics of maternal cigarette smoking during pregnancy and effects on child development. Dev Psychopathol 2012;24:1377-90.

4. Australian institute of health and welfare. Australia's mothers and babies 2013-in brief. Perinatal statistics series no. 31. Cat no. PER 72. Canberra: AlHW 2015.

5. Wills RA, Coory MD. Effect of smoking among Indigenous and nonIndigenous mothers on preterm birth and full-term low birthweight. Med J Aust 2008;189:490-4.

6. Passmore E, McGuire R, Correll P, et al. Demographic factors associated with smoking cessation during pregnancy in New South Wales, Australia, 2000-2011. BMC Public Health 2015;15:398.

7. Gould GS, Munn J, Watters T, et al. Knowledge and views about maternal tobacco smoking and barriers for cessation in Aboriginal and Torres Strait Islanders: a systematic review and metaethnography. Nicotine Tob Res 2013;15:863-74.

8. Passey ME, Bryant J, Hall AE, et al. How will we close the gap in smoking rates for pregnant Indigenous women? Med J Aust 2013;199:39-41.

9. Gould GS, Cadet-James Y, Clough AR. Getting over the shock: taking action on Indigenous maternal smoking. Aust $J$ Prim Health 2016;22:276.

10. Passey ME, D'Este CA, Stirling JM, et al. Factors associated with antenatal smoking among Aboriginal and Torres Strait Islander women in two jurisdictions. Drug Alcohol Rev 2012;31:608-16
11. Witte K, Cameron KA, McKeon JK, et al. Predicting risk behaviors: development and validation of a diagnostic scale. J Health Commun 1996;1:317-42.

12. Wong NC, Cappella JN. Antismoking threat and efficacy appeals: effects on smoking cessation intentions for smokers with low and high readiness to quit. J App/ Commun Res 2009;37:1-20.

13. Witte K, Allen M. A meta-analysis of fear appeals: implications for effective public health campaigns. Health Educ Behav 2000;27:591-615.

14. Gould GS, Watt K, Cadet-James Y, et al. Using the risk behaviour diagnosis scale to understand Australian Aboriginal smoking - A cross-sectional validation survey in regional New South Wales. Prev Med Rep 2015;2:4-9.

15. Witte K. Fear control and danger control: a test of the extended paralle process model (EPPM) Communication monographs. 1994;61:113-34.

16. Gould GS, Watt K, McEwen A, et al. Validation of risk assessment scales and predictors of intentions to quit smoking in Australian Aboriginal and Torres Strait Islander peoples: a cross-sectional survey protocol. BMJ Open 2014;4:e004887.

17. Popova L. The extended parallel process model: illuminating the gaps in research. Health Educ Behav 2012;39:455-73.

18. Rimal RN, Real K. Perceived risk and efficacy beliefs as motivators of change. Hum Commun Res 2003;29:370-99.

19. Tavakol M, Dennick R. Making sense of Cronbach's alpha. Int J Med Educ 2011;2:53-5.

20. Ingall G, Cropley M. Exploring the barriers of quitting smoking during pregnancy: a systematic review of qualitative studies. Women Birth 2010;23:45-52.

21. Gould GS, Patten C, Glover M, et al. Smoking in pregnancy among Indigenous women in high-income countries: a narrative review. Nicotine Tob Res 2017;19:506-17.

22. Flemming K, McCaughan D, Angus K, et al. Qualitative systematic review: barriers and facilitators to smoking cessation experienced by women in pregnancy and following childbirth. $J$ Adv Nurs 2015;71:1210-26.

23. Wigginton B, Lafrance MN. 'I think he is immune to all the smoke I gave him': how women account for the harm of smoking during pregnancy. Health Risk Soc 2014;16:530-46.

24. Gould GS, Munn J, Avuri S, et al. "Nobody smokes in the house if there's a new baby in it": Aboriginal perspectives on tobacco smoking in pregnancy and in the household in regional NSW Australia. Women Birth 2013;26:246-53.

25. Gould GS, Bittoun R, Clarke MJ. A pragmatic guide for smoking cessation counselling and the initiation of nicotine replacement therapy for pregnant Aboriginal and Torres Strait Islander smokers. J Smok Cessat 2015;10:96-105.

26. Witte K, Meyer G, Martell D. Effective health risk messages: a stepby-step guide. Thousand Oaks, CA: Sage Publications, 2001. 